\title{
Emancipación juvenil a través de la formación de la pareja. Una comparación entre los censos de 1991 y de 2001*
}

\author{
Pau Miret Gamundi \\ Universitat Autònoma de Barcelona. Departament de Geografia \\ Centre d'Estudis Demogràfics \\ pau.miret@uab.cat
}

Recibido: 10-10-2007

Aceptado: 25-06-2008

\section{Resumen}

Se busca analizar la emancipación familiar a través de la pareja en España en dos momentos en el tiempo: 1991 y 2001. La fuente de datos la componen los ficheros de microdatos de los censos de población. El indicador que conforma la variable dependiente es la relación entre el volumen de personas que conviven con su pareja y las que residen sin pareja con sus padres. Las variables de control son el momento de observación y el sexo. Las covariables independientes son la edad, la relación con la actividad y el nivel de instrucción. La técnica estadística utilizada es la regresión logística.

Palabras clave: modelos de emancipación, familia, parejas, España.

Abstract. Youth Emancipation through Partnership Constitution. A Comparison between the Census Results of 1991 and 2001

The goal of this paper is to analyse family formation through partnership constitution in Spain at two cross-sectional points in time, that is, 1991 and 2001. The data sources are microdata files from the population censuses. The ratio between living with a partner and living without a partner with parents is the dependent variable. Analysis is conducted for different years of observation and for both sexes: We fit a model for each of these groups. Age, labour force participation (activity status) and educational attainment are the independent variables. The statistical method applied is logistic regression which allows the separation of net effects for each independent variable.

Key words: leaving home models, family, partnership, Spain.

* Este trabajo se ha beneficiado de las ayudas a los proyectos de investigación del Ministerio de Ciencia y Tecnología para el estudio titulado Migraciones internas, constitución familiar y empleo: dinámicas temporales y territoriales (ref. SEJ2004-01534) y también para el estudio titulado Juventud, género e inmigración ante la inserción en el mercado laboral en España. ¿Substitución o complementariedad? ¿Efecto edad o efecto cohorte? (ref. SEJ2007-67569). 


\section{Sumario}

$\begin{aligned} \text { Datos observados, problemas planteados } & \text { Resultados } \\ \text { Paradigmas explicativos } & \text { Conclusiones } \\ \text { Metodología técnica } & \text { Referencias bibliográficas }\end{aligned}$

\section{Datos observados, problemas planteados}

Las proporciones de jóvenes que conviven con su pareja han sufrido en España un descenso sustantivo en comparación con otros países occidentales (Miret, 2003). Esta caída ha corrido paralela al incremento inusitado en el número de jóvenes que residen con sus padres sin haber formado una unión. Aquí, se va a circunscribir la "juventud» a un rango determinado de edades, aunque es complicado establecer hoy en día qué se entiende por tal. La consideración en este artículo del período juvenil se iniciará a los 20 años (un inicio algo tardío, quizá pensarán algunos, pero es la edad en que las situaciones de convivencia en pareja comienzan a ser significativas) y será generosa en su final, pues alcanzará los 39 años de edad (algo excesivo, tal vez piensen otros, pero corren tiempos de extensión de las etapas vitales, no sólo de la juventud).

Ultimamente, estos cambios sociales se pueden estudiar a través de la información proporcionada por los ficheros de microdatos de los censos de población, facilitados por el Instituto Nacional de Estadística (INE), una vez han sido convenientemente anonimizados ${ }^{1}$. Así, el censo de 1991 fue el primero en el cual, además del estado civil de las personas que convivían en un mismo hogar, se recolectó si un individuo se encontraba inserto en una relación de pareja. La variable ha vuelto a repetirse para el censo de 2001. La pura descripción de los datos nos informa que, en 1991, un 53,9\% de la población de entre 20 y 39 años convivía en pareja. De cómo han cambiado las cosas en una década nos informa el censo de 2001: entonces convivía en pareja un $41,7 \%$ de la población de entre 20 y 39 años, es decir, doce puntos porcentuales menos que diez años antes, de ahí que se hable de «crisis en la formación de uniones» (tal y como se ha puesto de manifiesto en otros análisis sobre el tema; véase Miret y Cabré, 2004, y Miret, 2007).

Obviamente, el tipo de hogar de quien no convivía en pareja ${ }^{2}$ dibujaba una realidad bien distinta según se tratara de población que aún convivía con sus padres o de gente que residía sola o en grupos de amigos sin estar vinculados a un núcleo familiar. Mientras que, en el primer caso, se trataba de indi-

1. Se trata de datos primarios, es decir, no se ha utilizado ninguna tabulación ofrecida por el INE en su página web, sino que se ha trabajado directamente sobre la muestra de los censos de población.

2. Quisiéramos remarcar que, a lo largo de este artículo, estudiamos conjuntamente a las parejas de hecho y a las de derecho, es decir, la pareja aquí tratada es tanto la matrimonial como la unión consensual, sin distinción. 
viduos familiarmente dependientes, en el segundo eran personas que podían considerarse emancipadas. En este sentido, los valores hablan por sí mismos. En efecto, del 46,1\% de los que en 1991 tenían entre 20 y 39 años y no convivían en pareja, un contundente $94,3 \%$ estaba aún con sus padres, un substantivo 4,7\% vivía en soledad y un nimio 1,0\% lo hacía en grupos no familiares. Diez años después, en 2001, las cosas habían cambiado, ciertamente, aunque no de manera radical: el $58,1 \%$ de la población de 20 a 39 años no residía en pareja, un $86,7 \%$ de este colectivo convivía con sus padres (una caída de este indicador de casi ocho puntos porcentuales), un 9,5\% residía en hogares unipersonales (casi cinco puntos más) y un $4,1 \%$ lo hacía en grupos no familiares (una situación prácticamente inexistente diez años atrás y que entonces tenía ya una evidente presencia social).

Otras cosas también han variado, incluso con mayor virulencia. Por ejemplo, la realidad social respecto a la división en la ocupación laboral entre los miembros de la pareja en España ha cambiado de manera substancial. En 1991, entre el total de parejas algún miembro de la cual tenía entre 20 y 39 años, en un $32,2 \%$ ambos componentes estaban laboralmente ocupados (una aproximación a lo que se denomina parejas de doble ingreso), pero en un $59,7 \%$ sólo lo estaba uno de ellos (el «cabeza de familia») y en el 8,1\% restante no lo estaba ninguno (pues ambos eran estudiantes o estaban en situación de desempleo). Diez años más tarde, en 2001, las parejas con un miembro que tenía entre 20 y 39 años se distribuían mitad y mitad entre aquéllas en que ambos estaban ocupados $(46,1 \%)$ y aquéllas en que sólo lo estaba uno de sus componentes $(47,2 \%)$, con un $6,7 \%$ en que ambos estaban inactivos o desocupados. En definitiva, entre las parejas jóvenes, el modelo de doble ocupación se había incrementado en catorce puntos porcentuales, con lo cual disminuía el de la división social del trabajo interior i exterior en el hogar en casi la misma cantidad. Pero aún se estaba muy lejos de alcanzar un modelo de familia igualitaria entre sexos, y el modelo complementario continuaba siendo en España, a principios del siglo XXI, mayoritario entre las parejas jóvenes. Además, aunque no será posible tratarlo con los datos censales, son muchas las investigaciones que enseñan que también se ha dado un cambio en la repartición de las tareas domésticas en el seno de la pareja (Meil, 1999; Durán, 2000).

\section{Paradigmas explicativos}

La intención de este artículo es descubrir si alguna de las razones que se apuntan como responsables de la caída de la nupcialidad contiene elementos de verosimilitud suficientes, más allá de toda duda razonable, en el caso particular de España y comparando dos momentos en el tiempo: 1991 con 2001.

En general, el cambio en el modelo de pareja se ha presentado, en teoría sociológica, como una de las razones más poderosas en la explicación de la crisis de la nupcialidad. La división social del trabajo en el seno del matrimonio, en la que el marido era el proveedor material y la mujer era la cuidadora esencial, se presentaba como la base de la solidaridad orgánica que precisaba todo 
grupo social (Durkheim, 1967). Un modelo familiar basado en la estricta separación según género garantizaba la funcionalidad de la pareja como célula básica de la sociedad (Parsons, 1949). En este sentido, el matrimonio es un contrato que, en su diferenciación de roles internos según criterios de género, supone una dependencia económica por parte de la mujer y una dependencia respecto a los servicios domésticos por parte del marido. En definitiva, el matrimonio es una institución funcional debido a la dependencia mutua derivada de la especialización de cada uno de los cónyuges en aquello en lo que tiene una ventaja comparativa: un miembro en la producción de bienes y servicios domésticos y el otro en la producción de bienes y/o servicios para el mercado a cambio de un salario (Parson y Bales, 1955).

Según esta teoría, el varón ocupado y la mujer dedicada a las tareas del hogar deberían ser los individuos con mayor probabilidad de haber transitado desde la casa de los padres hasta la convivencia en pareja, y esta probabilidad sería menor cuanto más se apartara el comportamiento del individuo de este modelo «tradicional».

Esta teoría funcionalista ha sido fuertemente contestada. En primer lugar, se cuestiona que la especialización comporte necesariamente un aumento de capacidad adaptativa, pues la misma se desvela como prácticamente inexistente ante situaciones de crisis: ¿Qué pasa si el marido pierde el trabajo o vive un período de fuerte precariedad en el mismo? Además, la especialización redunda en una sobrecarga crónica en la actividad laboral de los varones y el subempleo de las mujeres (Oppenheimer y Lew, 1995). Según esta crítica, la caída de la nupcialidad no sería reflejo de una pérdida de atractivo del matrimonio, sino de un retraso en su formación derivado de una estrategia adaptativa a unas nuevas y duras condiciones sociales. Si así fuera, se debería apreciar que el matrimonio ha perdido fuelle entre los más jóvenes, pero no lo ha hecho en absoluto entre los jóvenes adultos.

Con todo, para la teoría del capital humano, el nivel de instrucción tiene una fuerza explicativa mucho mayor que la relación con la ocupación y la actividad. De hecho, siempre siguiendo esta corriente, mediante el grado educativo, se puede establecer cuál es el valor del individuo en el mercado laboral, por lo que se puede estimar qué es lo que pierde en el caso de no estar en actividad. Es lo que se denomina coste de oportunidad. Siguiendo las teorías de Durkheim y Parsons arriba expuestas, la teoría microeconómica afirma que el coste de oportunidad de una mujer de formar una pareja y, en consecuencia, dar inicio a una familia, es racionalmente inasumible para las más instruidas (Becker, 1981). En otras palabras, la traducción en renta de un título escolar es más alta cuanto más elevado sea el grado educativo. Formar una pareja, con la división sexual del trabajo como marco cultural, supone abandonar la carrera laboral o disminuir substancialmente su velocidad: una mujer estará dispuesta a dar este paso sólo si su grado de instrucción le pronostica un futuro en el mercado de trabajo no demasiado brillante. Siguiendo esta directiva, la mujer debería tener una menor probabilidad de convivir en pareja cuanto mayor fuera su nivel de instrucción. 
En definitiva, tres son las hipótesis explicativas, en cierta forma complementarias, que se busca contrastar en este artículo: 1) el descenso de la emancipación a través de la pareja es paralelo a la desaparición del modelo «tradicional» de división del trabajo según género en el seno del hogar (hombre garante del pan, mujer cuidadora); 2) este patrón de separación de roles continúa en gran medida vigente y sólo se ha dado un retraso en el calendario de la emancipación familiar, es decir, las personas se casan más tarde, pero, cuando lo hacen, responden a las mismas pautas que otrora, y 3) la mayor instrucción de la mujer supone un incremento paralelo en el coste de oportunidad de formar una pareja, por lo que es reticente a dar dicho paso.

\section{Metodología y técnica}

Como se ha comprobado, las situaciones de convivencia entre los jóvenes son diversas, pero aquí van a tratarse sólo las dos más abundantes, a saber, por un lado, el estar conviviendo en soltería (ampliamente entendida, es decir, sin pareja corresidente, se esté o no casado) y con alguno de los padres, y, por otro lado, el convivir con la pareja (estén o no los padres también presentes en el hogar de la pareja corresidente). Otras situaciones, como vivir solo o con un grupo de personas entre las cuales no se encuentra ni el padre o la madre ni la pareja, serán excluidas del análisis. El indicador así utilizado es un tanto especial, puesto que mide la fuerza de la emancipación familiar a través de la formación de la pareja (sin considerar a los emancipados por otras vías), contraponiendo a quienes convivían con una pareja a quienes residían sin pareja con sus padres.

En definitiva, la variable objeto de estudio será dicotómica, con una categoría si se vive en soltería con los padres y otra complementaria si se convive con la pareja. Por medio de la regresión logística (véase la descripción de esta técnica estadística en Jovell, 1995), se dirimirá qué distingue a los jóvenes que residen en una situación de los que conviven en la otra. Así, el indicador calculado es la ratio entre vivir en pareja y vivir sin pareja con los padres, y se verá cómo influyen en ello las tres variables explicativas utilizadas, a saber: 1) la edad (siempre con los límites de 20 y 39 años); 2) la relación con la actividad y ocupación, y, finalmente, 3) el nivel de instrucción.

Se construirá un modelo para cada año de observación y para cada sexo, por lo que se plantea, por un lado, un análisis independiente para hombres (tabla 1a) y mujeres (tabla 1b), y, por otro, se separa la observación para el censo de 1991 (tablas 2a) y para el de 2001 (tabla 2b). La regresión logística permite separar el efecto de cada variable independiente una vez han sido controladas las otras variables independientes utilizadas. Se trata de un procedimiento similar a la estandarización (véase Menacho, 2002). En definitiva, las tablas 1 y 2 presentan el efecto de las tres variables enunciadas (edad, actividad e instrucción) sobre la relación entre quienes convivían con su pareja y quienes lo hacían sólo con sus padres, separando a hombres y mujeres y analizando independientemente los datos de 1991 y los de 2001. 
Tabla 1a. Modelo explicativo en la ratio de vivir en pareja en vez de residir sin pareja en casa de los padres (España, 1991, hombres)

\begin{tabular}{|c|c|c|c|c|}
\hline & Casos & $\%$ & Ratio & Significación \\
\hline General & 286.077 & 100,00 & 0,29 & $* * *$ \\
\hline \multicolumn{5}{|l|}{ Edad } \\
\hline 20 & 16.704 & 5,84 & 0,04 & $* * *$ \\
\hline 21 & 16.193 & 5,66 & 0,06 & $* * *$ \\
\hline 22 & 16.010 & 5,60 & 0,10 & $* * *$ \\
\hline 23 & 16.174 & 5,65 & 0,15 & $* * *$ \\
\hline 24 & 15.853 & 5,54 & 0,23 & $* * *$ \\
\hline 25 & 15.737 & 5,50 & 0,35 & $* * *$ \\
\hline 26 & 15.900 & 5,56 & 0,51 & $* * *$ \\
\hline 27 & 15.407 & 5,39 & 0,75 & $* * *$ \\
\hline 28 & 14.498 & 5,07 & 1,04 & $* *$ \\
\hline 29 & 14.472 & 5,06 & 1,48 & $* * *$ \\
\hline 30 & 14.265 & 4,99 & 1,86 & $* * *$ \\
\hline 31 & 14.063 & 4,92 & 2,31 & $* * *$ \\
\hline 32 & 14.021 & 4,90 & 2,91 & $* * *$ \\
\hline 33 & 13.760 & 4,81 & 3,47 & $* * *$ \\
\hline 34 & 12.638 & 4,42 & 3,95 & $* * *$ \\
\hline 35 & 12.513 & 4,37 & 4,39 & $* * *$ \\
\hline 36 & 11.870 & 4,15 & 4,73 & $* * *$ \\
\hline 37 & 12.152 & 4,25 & 5,02 & $* * *$ \\
\hline 38 & 12.213 & 4,27 & 5,85 & $* * *$ \\
\hline 39 & 11.634 & 4,07 & 5,84 & $* * *$ \\
\hline \multicolumn{5}{|l|}{ Acividad } \\
\hline Estudiantes & 21.489 & 7,51 & 0,26 & $* * *$ \\
\hline Ocupados & 211.908 & 74,07 & 3,85 & $* * *$ \\
\hline Parados, primer empleo & 11.960 & 4,18 & 0,46 & $* * *$ \\
\hline Parados, han trabajado antes & 30.116 & 10,53 & 1,98 & $* * *$ \\
\hline Jubilados/Incapacitados & 4.866 & 1,70 & 0,56 & $* * *$ \\
\hline Tareas del hogar & 505 & 0,18 & 3,32 & $* * *$ \\
\hline Otra situación & 1.341 & 0,47 & 0,83 & $* * *$ \\
\hline Servicio militar & 3.892 & 1,36 & 0,71 & $* * *$ \\
\hline \multicolumn{5}{|l|}{ Instrucción } \\
\hline Analfabeto & 1.604 & 0,56 & 0,50 & $* * *$ \\
\hline Sin estudios & 17.436 & 6,11 & 1,21 & $* * *$ \\
\hline Primaria incompleta & 74.767 & 26,20 & 1,56 & $* * *$ \\
\hline Primaria completa & 77.182 & 27,05 & 1,36 & $* * *$ \\
\hline FPI & 18.862 & 6,61 & 1,41 & $* * *$ \\
\hline FPII & 16.909 & 5,93 & 1,06 & ** \\
\hline Bachillerato & 42.787 & 14,99 & 0,94 & $* * *$ \\
\hline Otras medias & 3.698 & 1,30 & 1,20 & $* * *$ \\
\hline Ingeniería técnica & 4.765 & 1,67 & 0,80 & $* * *$ \\
\hline Diplomatura & 9.336 & 3,27 & 0,92 & $* * *$ \\
\hline Ingeniería superior & 1.859 & 0,65 & 0,71 & $* * *$ \\
\hline Licenciatura & 13.211 & 4,63 & 0,67 & $* * *$ \\
\hline Otro nivel educación superior & 1.583 & 0,55 & 1,09 & ns. \\
\hline Postgrado & 1.379 & 0,48 & 1,28 & $* * *$ \\
\hline Doctorado & 699 & 0,24 & 0,94 & ns. \\
\hline
\end{tabular}

Fuente: elaboración propia a partir del censo de 1991.

Nota: ${ }^{* *} \mathrm{p}<0,01 ;{ }^{* *} \mathrm{p}<0,05 ;{ }^{*} \mathrm{p}<0,10$; ns: no significativo. 
Tabla 1b. Modelo explicativo en la ratio de vivir en pareja en vez de residir sin pareja en casa de los padres (España, 1991, mujeres)

\begin{tabular}{|c|c|c|c|c|}
\hline & Casos & $\%$ & Ratio & Significación \\
\hline General & 281.972 & 100,00 & 0,61 & $* * *$ \\
\hline \multicolumn{5}{|l|}{ Edad } \\
\hline 20 & 15.820 & 5,61 & 0,07 & $* * *$ \\
\hline 21 & 15.508 & 5,50 & 0,12 & $* * *$ \\
\hline 22 & 15.211 & 5,39 & 0,17 & $* * *$ \\
\hline 23 & 15.584 & 5,53 & 0,27 & $* * *$ \\
\hline 24 & 15.184 & 5,38 & 0,37 & $* * *$ \\
\hline 25 & 15.308 & 5,43 & 0,53 & $* * *$ \\
\hline 26 & 15.715 & 5,57 & 0,72 & $* * *$ \\
\hline 27 & 14.914 & 5,29 & 0,95 & $* * *$ \\
\hline 28 & 14.332 & 5,08 & 1,22 & $* * *$ \\
\hline 29 & 14.227 & 5,05 & 1,49 & $* * *$ \\
\hline 30 & 14.554 & 5,16 & 1,86 & $* * *$ \\
\hline 31 & 14.298 & 5,07 & 2,17 & $* * *$ \\
\hline 32 & 13.826 & 4,90 & 2,25 & $* * *$ \\
\hline 33 & 13.946 & 4,95 & 2,48 & $* * *$ \\
\hline 34 & 12.925 & 4,58 & 2,65 & $* * *$ \\
\hline 35 & 12.588 & 4,46 & 2,78 & $* * *$ \\
\hline 36 & 12.110 & 4,29 & 2,78 & $* * *$ \\
\hline 37 & 11.979 & 4,25 & 2,74 & $* * *$ \\
\hline 38 & 12.228 & 4,34 & 2,85 & $* * *$ \\
\hline 39 & 11.715 & 4,15 & 2,85 & $* * *$ \\
\hline \multicolumn{5}{|l|}{ Acividad } \\
\hline Estudiantes & 26.080 & 9,25 & 0,52 & $* * *$ \\
\hline Ocupadas & 110.873 & 39,32 & 1,51 & $* * *$ \\
\hline Paradas, primer empleo & 16.809 & 5,96 & 0,70 & $* * *$ \\
\hline Paradas, han trabajado antes & 31.406 & 11,14 & 2,18 & $* * *$ \\
\hline Jubiladas/Incapacitada & s2.834 & 1,01 & 0,20 & $* * *$ \\
\hline Tareas del hogar & 93.252 & 33,07 & 11,74 & $* * *$ \\
\hline Otra situación & 718 & 0,25 & 0,36 & $* * *$ \\
\hline \multicolumn{5}{|l|}{ Instrucción } \\
\hline Analfabeta & 1.955 & 0,69 & 0,59 & $* * *$ \\
\hline Sin estudios & 18.982 & 6,74 & 1,41 & $* * *$ \\
\hline Primaria incompleta & 74.440 & 26,44 & 1,79 & $* * *$ \\
\hline Primaria completa & 73.762 & 26,20 & 1,48 & $* * *$ \\
\hline FPI & 17.122 & 6,08 & 1,56 & $* * *$ \\
\hline FPII & 10.577 & 3,76 & 1,04 & ns. \\
\hline Bachillerato & 41.618 & 14,78 & 1,06 & **** \\
\hline Otras medias & 5.505 & 1,96 & 0,98 & ns. \\
\hline Ingeniería técnica & 1.248 & 0,44 & 0,90 & * \\
\hline Diplomatura & 18.355 & 6,52 & 1,02 & ns. \\
\hline Ingeniería superior & 229 & 0,08 & 0,63 & $* * *$ \\
\hline Licenciatura & 15.457 & 5,49 & 0,71 & $* * *$ \\
\hline Otro nivel educación superior & 1.331 & 0,47 & 0,79 & $* * *$ \\
\hline Postgrado & 940 & 0,33 & 0,97 & ns. \\
\hline Doctorado & 451 & 0,16 & 0,86 & ns. \\
\hline
\end{tabular}

Fuente: elaboración propia a partir del censo de 1991.

Nota: ${ }^{* * *} \mathrm{p}<0,01 ;{ }^{* *} \mathrm{p}<0,05 ;{ }^{*} \mathrm{p}<0,10$; ns: no significativo. 
Tabla 2a. Modelo explicativo en la ratio de vivir en pareja en vez de residir sin pareja en casa de los padres (España, 2001, hombres)

\begin{tabular}{|c|c|c|c|c|}
\hline & Casos & $\%$ & Ratio & Significación \\
\hline General & 307.334 & 100,00 & 0,23 & $* * *$ \\
\hline \multicolumn{5}{|l|}{ Edad } \\
\hline 20 & 14.282 & 4,65 & 0,04 & $* * *$ \\
\hline 21 & 14.847 & 4,83 & 0,06 & $* * *$ \\
\hline 22 & 15.162 & 4,93 & 0,10 & $* * *$ \\
\hline 23 & 16.069 & 5,23 & 0,13 & $* * *$ \\
\hline 24 & 16.183 & 5,27 & 0,21 & $* * *$ \\
\hline 25 & 16.737 & 5,45 & 0,31 & $* * *$ \\
\hline 26 & 16.396 & 5,33 & 0,47 & $* * *$ \\
\hline 27 & 15.926 & 5,18 & 0,66 & $* * *$ \\
\hline 28 & 15.618 & 5,08 & 0,96 & $* *$ \\
\hline 29 & 15.814 & 5,15 & 1,30 & $* * *$ \\
\hline 30 & 15.178 & 4,94 & 1,81 & $* * *$ \\
\hline 31 & 15.091 & 4,91 & 2,27 & $* * *$ \\
\hline 32 & 14.968 & 4,87 & 2,85 & $* * *$ \\
\hline 33 & 15.171 & 4,94 & 3,34 & $* * *$ \\
\hline 34 & 15.242 & 4,96 & 4,04 & $* * *$ \\
\hline 35 & 14.952 & 4,87 & 4,82 & $* * *$ \\
\hline 36 & 15.212 & 4,95 & 5,45 & $* * *$ \\
\hline 37 & 15.568 & 5,07 & 6,14 & $* * *$ \\
\hline 38 & 14.845 & 4,83 & 6,55 & $* * *$ \\
\hline 39 & 14.073 & 4,58 & 7,71 & $* * *$ \\
\hline \multicolumn{5}{|l|}{ Acividad } \\
\hline Estudiantes & 53.120 & 17,28 & 1,34 & $* * *$ \\
\hline Otra situación & 4.453 & 1,45 & 0,93 & $* *$ \\
\hline \multicolumn{5}{|l|}{ Instrucción } \\
\hline Analfabetos & 1.888 & 0,61 & 0,55 & $* * *$ \\
\hline Sin estudios & 8.268 & 2,69 & 1,09 & $* * *$ \\
\hline Primaria incompleta & 41.986 & 13,66 & 1,44 & $* * *$ \\
\hline Primaria completa & 100.238 & 32,62 & 1,35 & $* * *$ \\
\hline FP grado medio & 22.919 & 7,46 & 1,47 & $* * *$ \\
\hline FP grado superior & 28.968 & 9,43 & 1,14 & $* * *$ \\
\hline Bachillerato & 49.673 & 16,16 & 0,96 & $* *$ \\
\hline Diplomatura & 25.019 & 8,14 & 0,77 & $* * *$ \\
\hline Licenciatura & 26.808 & 8,72 & 0,74 & $* * *$ \\
\hline Doctorado & 1.567 & 0,51 & 0,93 & ns. \\
\hline
\end{tabular}

Fuente: elaboración propia a partir del censo de 2001.

Nota: ${ }^{* * *} \mathrm{p}<0,01 ;{ }^{* *} \mathrm{p}<0,05 ;{ }^{*} \mathrm{p}<0,10$; ns: no significativo. 
Tabla 2b. Modelo explicativo en la ratio de vivir en pareja en vez de residir sin pareja en casa de los padres (España, 2001, mujeres)

\begin{tabular}{|c|c|c|c|c|}
\hline General & Casos & $\%$ & Ratio & Significación $_{* * *}$ \\
\hline \multirow{2}{*}{\multicolumn{5}{|c|}{ Edad }} \\
\hline $\begin{array}{l}\text { Edad } \\
20\end{array}$ & 13.522 & 4,42 & & *** \\
\hline 21 & 14.289 & 4,67 & 0,11 & $* * *$ \\
\hline 22 & 14.783 & 4,83 & 0,16 & $* * *$ \\
\hline 23 & 15.515 & 5,07 & 0,22 & $* * *$ \\
\hline 24 & 15.918 & 5,20 & 0,30 & $* * *$ \\
\hline 25 & 16.288 & 5,32 & 0,43 & $* * *$ \\
\hline 26 & 15.972 & 5,22 & 0,57 & $* * *$ \\
\hline 27 & 15.714 & 5,13 & 0,79 & $* * *$ \\
\hline 28 & 15.565 & 5,09 & 1,05 & $* * *$ \\
\hline 29 & 15.440 & 5,04 & 1,41 & $* * *$ \\
\hline 30 & 15.245 & 4,98 & 1,72 & $* * *$ \\
\hline 31 & 15.115 & 4,94 & 2,03 & $* * *$ \\
\hline 32 & 15.274 & 4,99 & 2,41 & $* * *$ \\
\hline 33 & 15.475 & 5,06 & 2,75 & $* * *$ \\
\hline 34 & 15.587 & 5,09 & 3,06 & $* * *$ \\
\hline 35 & 15.121 & 4,94 & 3,17 & $* * *$ \\
\hline 36 & 15.501 & 5,06 & 3,40 & $* * *$ \\
\hline 37 & 15.890 & 5,19 & 3,56 & $* * *$ \\
\hline 38 & 15.259 & 4,99 & 3,73 & $* * *$ \\
\hline 39 & 14.610 & 4,77 & 3,80 & $* * *$ \\
\hline \multicolumn{5}{|l|}{ Acividad } \\
\hline Estudiantes & 61.988 & 20,25 & 0,65 & $* * *$ \\
\hline Ocupadas & 145.838 & 47,65 & 1,23 & $* * *$ \\
\hline Paradas, primer empleo & 8.951 & 2,92 & 0,62 & $* * *$ \\
\hline Paradas, han trabajado antes & 30.177 & 9,86 & 1,37 & $* * *$ \\
\hline Jubiladas/Incapacitadas & 2.458 & 0,80 & 0,25 & $* * *$ \\
\hline Tareas del hogar & 52.471 & 17,14 & 7,00 & $* * *$ \\
\hline Otra situación & 4.200 & 1,37 & 0,84 & $* * *$ \\
\hline \multicolumn{5}{|l|}{ Instrucción } \\
\hline Analfabetas & 1.837 & 0,60 & 0,62 & $* * *$ \\
\hline Sin estudios & 6.864 & 2,24 & 1,13 & $* * *$ \\
\hline Primaria incompleta & 32.800 & 10,72 & 1,42 & $* * *$ \\
\hline Primaria completa & 86.875 & 28,38 & 1,44 & $* * *$ \\
\hline FP grado medio & 22.365 & 7,31 & 1,36 & $* * *$ \\
\hline FP grado superior & 27.398 & 8,95 & 1,12 & $* * *$ \\
\hline Bachillerato & 52.925 & 17,29 & 1,00 & ns. \\
\hline Diplomatura & 39.049 & 12,76 & 0,84 & $* * *$ \\
\hline Licenciatura & 34.402 & 11,24 & 0,72 & $* * *$ \\
\hline Doctorado & 1.568 & 0,51 & 0,76 & $* * *$ \\
\hline
\end{tabular}

Fuente: elaboración propia a partir del censo de 2001.

Nota: ${ }^{* * *} \mathrm{p}<0,01 ;{ }^{* *} \mathrm{p}<0,05{ }^{*} \mathrm{p}<0,10$; ns: no significativo. 
Las primeras dos columnas de las tablas muestran, respectivamente, el número de casos (la frecuencia en cada categoría) y el porcentaje sobre el total para cada variable (es decir, la estructura para cada variable), la tercera columna ofrece la ratio de población en pareja respecto a la población sin pareja conviviendo con los padres (el objeto de estudio) y la cuarta, la significación estadística de cada estimación realizada (hasta qué punto podemos afirmar que una categoría presenta una ratio estadísticamente diferente a la población en general). La edad ha sido considerada de manera discreta, es decir, edad a edad, pues así lo permite la información censal. Para la relación con la actividad y el nivel de instrucción, se utilizan las categorías censales, las cuales, en general, coinciden entre el censo de 1991 y 2001, con algunas pequeñas diferencias que se harán notar a lo largo del artículo.

La muestra de 1991 se compone de 286.077 hombres y de 281.972 mujeres, y la de 2001, de 307.334 hombres y de 306.083 mujeres, enmarcados entre los 20 y los 39 años de edad. Unas excelsas cantidades que dotan de una gran fiabilidad y validez al análisis que a continuación se ofrece.

\section{Resultados}

Si se calcula directamente sin más la relación según sexo entre los que convivían en pareja sobre los que convivían con los padres, se ve que, en 1991, era de 1,00 entre los hombres (es decir, ambas situaciones de convivencia estaban igualadas) y de 1,54 entre las mujeres (es decir, entre las mujeres de entre 20 y 49 años había 154 mujeres conviviendo con su pareja por cada 100 que residían sólo con sus padres: las emancipadas mediante formación de la pareja eran un $54 \%$ más que las no emancipadas). La observación diez años más tarde, en 2001, ofrece unos indicadores de 0,68 y 1,00 respectivamente para hombres y mujeres: 68 varones de entre 20 y 39 años vivían con su pareja por cada 100 solteros y residiendo con sus padres, una ratio que, entre las mujeres, era de la mitad en cada situación. Así, la caída en la ratio de emancipación familiar fue de algo más de 30 puntos porcentuales en los hombres y de más de 50 puntos porcentuales en las mujeres.

Pero estas cifras «brutas» estaban fuertemente influidas por la estructura por edad de la población de entre 20 y 39 años, por su actividad laboral y por su nivel de instrucción (entre otras posibles interferencias que no incluiremos), tal y como se demuestra si extraemos de estos indicadores el efecto «neto» de la edad, la actividad y la instrucción (sin interferencias entre una y otra variable), procedimiento técnico que recibe el término genérico de «estandarización».

En efecto, la ratio estandarizada por estas variables y debidas en exclusiva al sexo y al año de observación (las dos variables de control), y no a la estructura y a la pauta de conducta por edad, por actividad o por nivel de instrucción, fue en 1991 de 0,29 para los hombres y de 0,61 para las mujeres (tabla 1), y en 2001 (tabla 2) había descendido ligeramente para los hombres $(0,23)$ y prácticamente no había variado para las mujeres, con una ratio de 0,60 (una minús- 
cula caída de una centésima). En definitiva, podemos convenir que prácticamente toda la explicación de los cambios observados en la emancipación con formación de la pareja entre 1991 y 2001 radica en la variación de la estructura y del comportamiento según edad, nivel de la relación con la actividad y nivel de instrucción.

En general, tanto la diferencia entre momentos temporales como entre sexos se ha suavizado de manera substancial. Ciertamente, dentro del rango de edad 20-39 años, por un lado, convivir con la pareja en vez de hacerlo con los padres fue siempre más frecuente entre las mujeres que entre los hombres (con una diferencia estandarizada en la ratio entre sexos de poco más de tres décimas, en ambos momentos observados) y, por otro lado, tanto para ellos como para ellas, esta ratio fue un poco menor en 2001 que en 1991 (con una diferencia estandarizada en la ratio de seis centésimas en los varones y una centésima en las mujeres). Todas las demás diferencias observadas entre momentos y entre sexos fueron causadas por una o por diversas de las variables independientes incluidas en el modelo explicativo.

A partir de ahora, se denominará al indicador que constituye la variable dependiente como "ratio de emancipación familiar a través de la pareja» y se anotará para abreviar como REP. Nuestro objetivo a continuación es mostrar como influenciaron la edad, la actividad y el nivel de instrucción en la suavidad de la caída en la REP neta entre 1991 y 2001 (al contrario de la REP observada) y porque la diferencia fue tan menuda entre sexos, una vez se han aplicado técnicas de extracción de los efectos netos.

\section{La pauta por edad}

Evidentemente, convivir con la pareja en vez de con los padres estaba intrínsecamente relacionado con la edad: cuanto más alejado se estuviera de los 20 años y, en consecuencia, más cercano a los 39, mayor era la probabilidad de estar conviviendo con la pareja y, complementariamente, menor la de estar conviviendo sólo con los padres. Queremos destacar, sin embargo, que los indicadores que ahora se presentan ofrecen la influencia «neta» de la edad, es decir, una vez se han eliminado los efectos de las otras dos variables incluidas en el modelo (gráfico 1). Con estas premisas, podemos afirmar que la contribución de la edad en la probabilidad de estar conviviendo en pareja y no en soltería con los padres se mantuvo constante hasta los 31 años entre 1991 y 2001 (independientemente de la situación de actividad e instrucción del individuo) y fue idéntico en ambos momentos para hombres y mujeres. En conclusión, los cambios temporales y las diferencias entre géneros en el efecto de la edad sobre la REP tiene sentido analizarlos únicamente más allá de los 31 años de edad.

Así, en primer lugar, se centrará la atención en cómo ha cambiado en el tiempo la influencia de la edad para los mayores y las mayores de 31 años en la REP, es decir, en la emancipación familiar a través de la formación de una unión, fuera ésta cohabitación o matrimonio. Para empezar, comprobamos que la edad es una variable mucho menos determinante para las mujeres que 


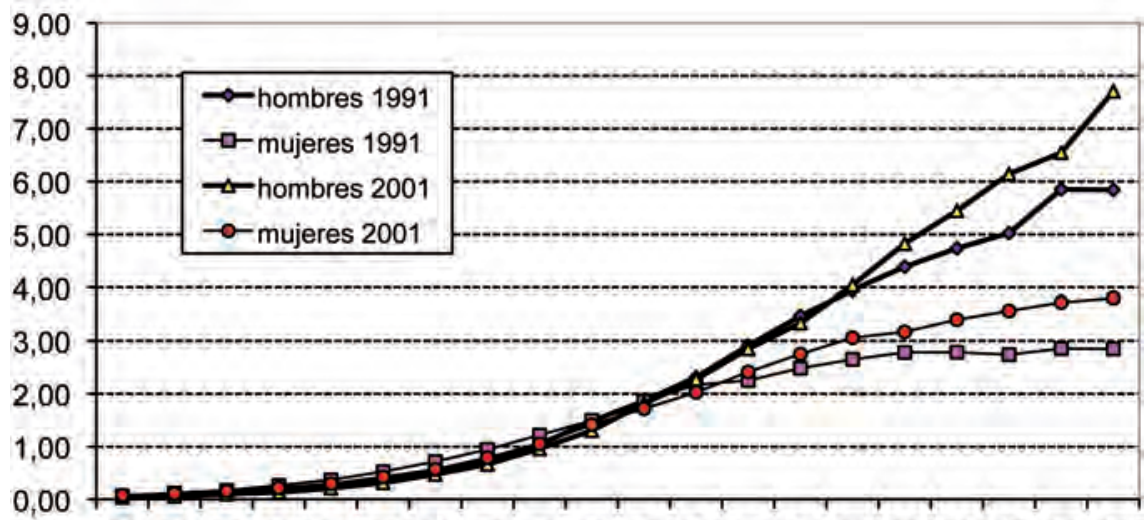

$\begin{array}{llllllllllllllllllll}20 & 21 & 22 & 23 & 24 & 25 & 26 & 27 & 28 & 29 & 30 & 31 & 32 & 33 & 34 & 35 & 36 & 37 & 38 & 39\end{array}$

Gráfico 1. Ratio de emancipación familiar a través de la pareja según edad, estandarizada por actividad e instrucción (España, 1991 y 2001, por sexos). Fuente: tablas 1 y 2.

para los hombres, aunque en ambos sexos su influencia se haya incrementado entre 1991 y 2001 en prácticamente la misma cuantía. Es decir, la transición desde la familia de origen a un núcleo conyugal propio tiene cada vez más que ver con el hecho de que "ya se tiene una edad", y la misma presiona de manera poderosa para dar el paso que supone la formación de la pareja.

En concreto, entre los hombres, la influencia de la edad para los menores de 34 años se mantuvo constante entre ambos momentos, pero de los 35 años en adelante, los varones en el año 2001 experimentaron una probabilidad de emanciparse a través de la pareja a causa de su edad significativamente superior a la registrada en 1991: a principios del siglo XXI, no haber aún formado una pareja pasados los 35 años (sic) ya pasaba de castaño oscuro. En contraste, entre las mujeres, el punto de corte fueron los 32 años, de manera que la REP antes de esa edad debido a la etapa biográfica que estaban atravesando fue algo superior (casi nada) en 1991 que en 2001, pero allende este punto etáneo, la diferencia fue de mayor importancia (gráfico 1). En conclusión, una vez controlado el efecto de la relación con la actividad y del nivel de instrucción, sólo entre los jóvenes más adultos el efecto de la edad en la emancipación juvenil a través de la pareja se incrementó en los diez años que separan 1991 de 2001, pero entre los más jóvenes no se apreció para nada una mayor presión en la edad para dejar de convivir con los padres y formar pareja: los menores de 35 años y las menores de 32 aún tenían toda la vida por delante para ello.

Este análisis debería complementarse con el estudio de las otras dos posibles situaciones de convivencia, a saber, vivir en soledad y con un grupo de amigos, para descubrir si este retraso en la emancipación a través de la pareja se vio compensado con una mayor autonomía a través de formas alternativas 
de hogar o únicamente supuso más dependencia familiar. Pero esto ya formará parte de otro artículo.

\section{El efecto de la relación con la actividad en la emancipación residencial a través de la formación de la pareja}

En la construcción de todo modelo interpretativo, deben distinguirse la estructura de la característica social estudiada y la ley que rige la conducta de cada grupo considerado. En el caso de la actividad, observamos, por un lado, la estructura de la población en su relación con el mercado de trabajo en cada momento y para cada sexo y, por otro lado, el comportamiento de cada categoría en relación con la actividad laboral. El modelo «clásico» en la relación entre convivir en pareja y la actividad laboral sería aquél que detectara, por un lado, que la mayoría de los varones analizados están trabajando y que ésta es la categoría que asume la mayor probabilidad de estar conviviendo en pareja en vez de estar en soltería residiendo con los padres. De manera complementaria, si los datos siguieran este modelo de estricta separación de roles, deberían también desvelar que lo más habitual entre las mujeres es dedicarse a las tareas del hogar (ser inactivas) y, entre ellas, la probabilidad de estar unida en pareja era mayor que en cualquier otra relación con la actividad.

La situación social de los hombres respondía diáfanamente a este modelo, tanto en 1991 como en 2001, aunque algunos puntos habían variado ligeramente entre uno y otro momento. Así, en 1991, tres cuartas partes de los hombres de entre 20 y 39 años estaban ocupados y la probabilidad que estos estuvieran en pareja era 3,5 veces superior a la pauta general para los varones en España (con una REP específica para los ocupados de 3,8 frente al 0,3 general). En 2001, el monto relativo de los que estaban trabajando en ese rango de edad había disminuido en cuatro puntos porcentuales (de manera que se situaba en un 70\%) y la fuerza de la REP había descendido hasta 2,3 (frente al 0,2 de la REP general estandarizada en ese momento); es decir, la posibilidad que un ocupado estuviera conviviendo en pareja en vez de vivir con sus padres era 2,1 veces superior a la REP de la población total de varones, independientemente de la edad que se tuviera y del nivel de instrucción alcanzado. En consecuencia, estar ocupado continuaba siendo la situación mayoritaria entre los hombres de entre 20 y 34 años en España, y la asociación entre convivir en pareja y tener un empleo era y es la más intensa entre los varones españoles. No obstante, mientras que la REP para un varón estándar apenas había variado entre 1991 y 2001, la asociación entre estar ocupado y conviviendo en pareja se había erosionado con el paso del tiempo, aunque aún brillaba refulgentemente en el amanecer del siglo XXI. Pero hay que observar la cara femenina del fenómeno antes de dictar un diagnóstico acertado sobre la influencia de la relación en el mercado de trabajo de los miembros potenciales de una pareja y la formación de una unión con convivencia en el mismo hogar.

Por otra parte, de acorde con ese modelo que hemos denominado clásico, la mayor probabilidad entre las mujeres de convivir en pareja y no residir sol- 
teras con sus padres era la dedicación a la economía doméstica, y aunque la REP para esta situación de actividad disminuyó considerablemente en diez años, aún presentaba en 2001 una contundente asociación. Así, mientras que en 1991 dedicarse a las tareas del hogar (tal y como lo hacía un tercio de las mujeres de entre 20 y 39 años) supuso una probabilidad de vivir en pareja en vez de con los padres doce veces superior a la pauta general, en 2001 (en que la proporción de mujeres "amas de casa" había disminuido a la mitad, a un $17 \%$ ), esta ratio era siete veces superior al de toda la población femenina. En conclusión, muchas menos mujeres se dedicaban íntegramente a la economía doméstica (aunque el quantum de las mismas aún era considerable a principios del siglo XXI), y la probabilidad de que las mismas fueran las que convivían en pareja también se desgastó poderosamente. Con todo, el modelo continuaba presente en la España del siglo XXI, aunque estuviera perdiendo fuelle a marchas forzadas.

En definitiva, el modelo «tradicional» de división del trabajo entre el ámbito productivo y el reproductivo gozaba de una mala salud de hierro, aunque es cierto que en España el desgaste del mismo ha corrido paralelo a la extensión biográfica en la etapa vital de convivencia con los padres durante la juventud y a la caída en la formación de parejas (Miret y Cabré, 2005). Sin embargo, es evidente que la dedicación en exclusiva de algún miembro de la pareja a la economía doméstica implica necesariamente que el otro sea el único proveedor de recursos financieros, puesto que la reproducción doméstica de momento nunca se ha asociado a un salario. En otras palabras, afirmar que el descenso en la formación de parejas se asocia a un descenso en la división social del trabajo es una explicación con altas dosis de tautología en su interior y, en consecuencia, no admisible científicamente.

Hay que anotar que, en el caso de los varones, también la dedicación a la economía doméstica estaba altamente relacionada con convivir en pareja en vez de con los padres, con una ratio casi al mismo nivel que la calculada para los que tenían un empleo. En concreto, entre los hombres, dedicarse a la reproducción tenía, en 1991, una probabilidad 3,3 veces superior de vivir en pareja que con los padres, una ratio que cayó a 2,3 en 2001. Con todo, la presencia de varones con tal función en la pareja era y es testimonial, de manera que pasó de representar a un 0,2\% de la población masculina en 1991 a representar un $0,3 \%$ en 2001 . Así, pues, ni por asomo se le estaba dando la vuelta a la tortilla.

En definitiva, sin lugar a dudas, el modelo en que uno de los miembros de la pareja se dedicaba en exclusiva a las tareas del hogar favorecía en alta medida la formación de parejas, y aunque lo hacía mucho más en el caso que fuera el miembro femenino de la pareja quien lo hiciera, no hay que despreciar la fuerza que tenía el que fuera el cónyuge masculino el dedicado al cuidado del hogar.

El censo de 1991 no permitió a dos personas del mismo sexo registrarse como pareja, por lo que los 505 varones que afirmaron dedicarse en exclusividad a tareas domésticas, si convivían en pareja, lo hacían (por imperativo legal) con una mujer, por lo que no puede comprobarse la hipótesis de que la dedicación a la economía reproductiva de los varones pudiera tener lugar fun- 
damentalmente entre parejas homosexuales, en que — se podría decir — uno de los miembros adoptase un papel de género femenino. Sin embargo, sí puede estudiarse esta situación en el censo de 2001, pues a las parejas homosexuales se les permitió declararse como tales, con lo cual quedó rotundamente desmentida la hipótesis que acaba de formularse: mientras que en un $28,7 \%$ de las parejas heterosexuales uno de los miembros se dedicaba a la economía doméstica, en las homosexuales este porcentaje era del 2,9\%.

Y así también, de manera complementaria, como síntoma del cambio en la sociedad española, se había incrementado notablemente la proporción de las mujeres que tenían un empleo en este rango de edades, pasando de casi un $40 \%$ en 1991 a casi la mitad de la población femenina de entre 20 y 39 años en 2001. En consecuencia, la disminución en quince puntos porcentuales de las que se dedicaban a la economía doméstica se explicaba en gran parte con el incremento en diez puntos en las ocupadas. Sin embargo, la propensión a convivir en pareja en vez de con los padres cuando la mujer trabajaba había disminuido con el tiempo: mientras la REP había sido de 1,5 en 1991 (entre las ocupadas, una vez las variables de edad y nivel de instrucción habían sido neutralizadas, por cada tres mujeres que convivían en pareja, dos lo hacían sin pareja y con sus padres), sólo alcanzó a ser de 1,2 en 2001. En definitiva, entre las ocupadas, de haber un $50 \%$ más de mujeres conviviendo en pareja que con sus padres en 1991 a causa de tener un empleo, se había pasado a un $20 \%$ más en 2001. La ocupación femenina continuaba siendo una característica importante en la emancipación residencial de la mujer a través de la pareja, pero no tanto como antes.

En España, dedicarse a estudiar durante la etapa vital de juventud ha sido y es una situación cada vez más extendida entre los jóvenes (véase si no, por ejemplo, el pormenorizado análisis en Baizán et al., 2002). Tras los datos censales, se observa que, entre los 20 y los 39 años, entre aquéllos que convivían o bien con sus padres o bien con su pareja, mientras que en 1991 eran estudiantes a tiempo completo un $7,5 \%$ de los varones y un $9,3 \%$ de las mujeres, en 2001 lo eran, respectivamente, un 17,3\% y un 20,3\%. En consecuencia, aumentó la dedicación al estudio para ambos sexos y se matunvo una estructura en que las mujeres estudiaban como actividad exclusiva en mayor proporción que los hombres.

Por otro lado, el modelo que se considera habitual (cuanto menos en las sociedades del sur de Europa) estipula que estudiar supone una exigua propensión a estar conviviendo con tu pareja en vez de estar con la familia de procedencia. Hay que acabar los estudios previamente a pensar en dejar de vivir con los padres y formar pareja. Y así, en principio, ocurría. En efecto, para los varones, en 1991, ésta era la probabilidad más baja: por cada 100 que vivían con sus padres, 26 convivían en pareja, es decir, entre los estudiantes a tiempo completo, la ratio era de 1 hombre en pareja por cada 4 con los padres. En contraste, entre las mujeres, la ratio fue, en 1991, de 0,5 (el doble que la masculina), es decir, entre las estudiantes, por cada mujer conviviendo en pareja, 2 estaban residiendo con sus padres. 
Esta ratio por motivo de estudios aumentó ligeramente para ellas, pues en 2001 llegó a ser de 0,6, pero reveló una auténtica revolución en el comportamiento masculino: ser estudiante a tiempo completo implicaba para ellos una probabilidad de estar en pareja un 34\% más alta que la de estar soltero conviviendo con los padres (la REP invirtió su sentido para los varones). En cierto sentido, la masculinidad había roto, en 2001, con la absoluta animadversión entre estudiar y la emancipación juvenil a través de la formación de la pareja propia de otros tiempos no muy lejanos (quien sabe si a través de una compañera que, con su trabajo, permitía al varón continuar estudiando). Sea como fuere, la variable género volvía a hacer acto de presencia, pues mientras los hombres estudiantes "podían» convivir en pareja, para las mujeres estudiar suponía un grave inconveniente para dejar de residir con los padres.

Una de las transiciones entre la juventud y el mundo adulto es la entrada en actividad y la subsiguiente consecución del primer empleo. La información censal permite distinguir a quienes estaban a la espera de encontrar un primer trabajo. Como cabía esperar, esta situación estuvo asociada con una mayor probabilidad de continuar conviviendo con los padres en vez de hacerlo en pareja, para ambos sexos y en ambos momentos en el tiempo. La mejora de las condiciones laborales entre los jóvenes quedó en evidencia ante el retroceso en la proporción de los que estaban en estas condiciones de búsqueda del primer empleo, pues ésta descendió a la mitad entre 1991 y 2001, puesto que bajó del 4 al 2\% entre los varones y del 6 al 3\% entre las mujeres (de nuevo, la estructura respecto a estas variables era algo más desfavorable entre el colectivo femenino).

Respecto al modelo, el efecto de esta categoría (los que se encontraban buscando un primer empleo) no varió ni un ápice entre los varones, para los cuales la probabilidad de estar conviviendo con los padres era el doble que la de estar conviviendo en pareja (con una REP de 0,5). En contraste, entre las mujeres, el indicador fue algo menor en 2001 que en 1991: mientras que en este año por cada 7 mujeres que buscaban un primer empleo conviviendo en pareja había 10 en la misma situación de actividad residiendo solteras con sus padres, en 2001 la ratio era de 6 de cada 10. En definitiva, la situación de espera para entrar en el mercado de trabajo por primera vez era nefasta para la formación de parejas, muy en especial para los hombres, aunque la tendencia era que se igualara el efecto negativo para ambos sexos.

Pero mientras que la búsqueda del primer empleo tenía un efecto negativo en la emancipación residencial a través de la pareja, cuando ya se había entrado en el mercado laboral, el desempleo afectaba, en general, en sentido contrario. Aquí también se percibió la existencia, en 2001, de un modelo contrastado entre géneros, aunque tal distancia era una novedad, pues no había sido detectada diez años atrás. En efecto, en 1991, los desempleados que habían trabajado con anterioridad, independientemente del sexo, tenían el doble de probabilidad de estar conviviendo en pareja que con los padres. Muy probablemente, ello se debía a que se trataba de una situación laboral sobrevenida, puesto que se quedaban en paro con posterioridad a la emancipación residencial a través de la pareja. 
En contraste, en 2001, esta relación de probabilidades era de 1 para los varones (es decir, estaban los mismos en casa de sus padres que conviviendo en pareja), pero las mujeres paradas que habían trabajado tenían un 37\% más de probabilidades de estar en pareja que de vivir con los padres. En otras palabras, mientras que el efecto positivo del paro tras haber trabajado en la emancipación residencial con pareja se había desvanecido completamente entre los varones, continuaba estando asociado con esta transición de manera importante entre las mujeres. En definitiva, en este proceso, tener un empleo era imprescindible entre los hombres, pero mucho menos esencial entre las mujeres.

El estar realizando el servicio militar obligatorio sólo era posible en 1991 y para los varones, y así estaba un 1,4\% de la muestra observada entonces. En efecto, en 2001, estar en el ejército era una profesión a la que podían acceder tanto hombres como mujeres. Aunque, muy probablemente, la mayoría de los hombres que estaban en 1991 haciendo la «mili» residían en barracones y cuarteles, muchos de ellos debían ser recogidos en el censo como conviviendo aún con sus padres: con todo, por cada 7 que decían convivir en pareja, 10 quedaron censados con sus padres. Una distancia entre unos y otros no especialmente substancial.

Finalmente, se quisiera llamar la atención sobre la situación que, en general, estuvo más alejada de la transición desde la casa de los padres hasta la convivencia en pareja entre los jóvenes en España, a saber, el percibir una pensión (que a estas edades debía ser de invalidez). Las REP fueron, en 1991 y 2001, respectivamente, de 0,6 y 0,4 para los hombres y de 0,2 y 0,25 para las mujeres. La probabilidad de estar en pareja y no con los padres era mínima en esta situación de actividad.

\section{El nivel de instrucción y su función en la emancipación residencial a través de la pareja}

El nivel de instrucción de la población que tenía entre 20 y 39 años que o bien vivía en casa de los padres sin pareja o bien estaba conviviendo en pareja se incrementó notablemente entre 1991 y 2001, en especial entre las mujeres. Así, por ejemplo, mientras que, en 1991, un 60\% de esta submuestra tenía como máximo estudios primarios, en 2001, un 50\% de los varones y un $60 \%$ de las mujeres estaban por encima de este nivel.

En general, dentro del primer tramo de la carrera educativa (hasta completar los estudios obligatorios), una mayor instrucción supuso una mayor probabilidad de estar en pareja y no en soltería viviendo con los padres. La única acotación que debe hacerse a esta afirmación es que no importaba mucho para este indicador si los estudios primarios se habían o no completado con éxito (incluso podía afirmarse que, con la obligatoria incompleta, la emancipación era algo mayor que con este grado concluido).

Un nivel muy similar en la escala de esta ratio era el presentado por la población que había alcanzado desde un nivel de estudios primarios hasta una formación profesional de primer grado, también llamada en 2001 ciclo for- 
mativo de grado medio. En este grupo, se encontraba poco más del $30 \%$ del total de la muestra en 1991 y alrededor de un 40\% de los varones en 2001, así como un 35\% de las mujeres en este último año. Ésta es la estructura en lo que respecta a este específico nivel de instrucción, pero veamos ahora la pauta de comportamiento registrada: tanto hombres como mujeres en este punto de la escala educativa tenían alrededor de un $40 \%$ más de probabilidades (con respecto al total de la población de cada sexo) de estar conviviendo en pareja en lugar de estar residiendo con sus padres. En definitiva, este nivel básico de estudios era, a la vez, en el que estaban más jóvenes (en especial, con unos estudios puramente obligatorios) y en el que la propensión a la emancipación familiar a través de la pareja era mayor. Sin duda, como ahora veremos, un mayor nivel de estudios estaba asociado a una prolongación de la dependencia en casa de los padres o, si queremos ver la otra cara de la moneda, a una espera más larga hasta la transición de formar pareja.

Así, la REP era algo inferior para la población que tenía estudios medios, para la cual la probabilidad de estar en pareja estaba igualada con la de vivir solteros en casa de los padres. En esta condición educativa se encontraban poco más del 20\% en 1991 y poco más del $25 \%$ en 2001. Quisiéramos remarcar que, con respecto a los estudios medios, entre hombres y mujeres ni existía diferencia en la estructura, ni ofrecían un comportamiento diferencial con respecto a la relación de su nivel de instrucción y la emancipación residencial a través de la formación de la pareja.

Los niveles de instrucción de educación superior eran más minoritarios a medida que se elevaba en la escala educativa y, además, a mayor grado, mayor era la probabilidad de estar aún con los padres y en soltería y no haber formado un núcleo conyugal o una familia propia. Así, en 1991, un 5\% de los hombres y un 7\% de las mujeres tenían una diplomatura o una ingeniería técnica, porcentaje que ascendió en 2001 a un 8 y un 13\%, respectivamente; por otro lado, estos valores de estructura para la ingeniería superior o la licenciatura eran del 6\% para los hombres y las mujeres en 1991, y de un 9 y un $11 \%$, respectivamente, en 2001. Por otro lado, la REP en la diplomatura o equivalente fue de 0,8 y en la ingeniería de 0,7 , es decir, por cada 10 individuos en cada nivel de estudios que todavía convivían con sus padres, convivían con su pareja 8 jóvenes entre los diplomados y 7 entre los licenciados.

En conclusión, para ambos sexos y sin cambios entre 1991 y 2001, una vez se había superado la educación primaria, una mayor educación estaba claramente asociada a una menor probabilidad de haber transitado de casa de los padres a la convivencia en pareja.

\section{Conclusiones}

El proceso de dejar de convivir con los padres y constituir un núcleo conyugal se retrasó de manera importante entre 1991 y 2001, pero una cosa diferente es el peso que los años de vida tenían en la transición entre uno y otro estado: así, esta presión para formar pareja se mantuvo estable en ambos momentos 
entre los 20 y los 31 años, y fue idéntica para ambos sexos. Sin embargo, a partir de los 31 años, el efecto de la edad era más intenso para los hombres que para las mujeres, y ha sido más fuerte en 2001 que en 1991, es decir, la probabilidad de dejar de convivir con los padres y formar pareja para los jóvenes adultos, una vez se había neutralizado el efecto de la actividad y de la educación, se cernía sobre los varones con mayor insidia que sobre las mujeres, y se apreció de forma más aguda en 2001 que en 1991 (siempre considerando únicamente a los hombres mayores de 34 años y a las mujeres mayores de 32 años). Ya era hora a estas tardías edades que los jóvenes y las jóvenes dejaran de vivir con los padres y formaran pareja.

En relación con la actividad laboral, el modelo masculino en la emancipación residencial a través de la pareja ha sufrido cambios espectaculares y mucho más visibles que en el caso femenino. Por un lado, ser estudiante a tiempo completo, que en 1991 era la situación menos favorable a este proceso, ha perdido toda su negatividad y se ha transformado, en 2001, en una posición para los varones que no rehuye formar una unión, sino todo lo contrario. En cambio, los hombres que estaban en 1991 en paro tras haber trabajado con anterioridad tenían el doble de probabilidades de estar en pareja en vez de continuar viviendo con los padres, pero, en 2001, esta situación de actividad estaba asociada de igual manera a convivir con los padres que a convivir con la pareja.

En contraste, el modelo femenino en lo que respecta a la asociación entre actividad y formación de la pareja apenas había variado entre los dos momentos estudiados, a pesar del cambio acaecido - eso sí- en la estructura laboral de las mujeres observadas. Pero siempre hay que distinguir con claridad los cambios en la estructura de los acometidos en la pauta de conducta, y si bien es cierto que el número de mujeres amas de casa se ha reducido de manera importante para el grupo de edad estudiado y se ha incrementado la cantidad de ocupadas, la probabilidad de emanciparse y formar pareja se ha mantenido bastante estable para unas y otras, de manera que es extraordinariamente alta para las primeras y también significativamente superior a la pauta general para éstas últimas.

Finalmente, en relación con el nivel de instrucción, vemos que, en la actualidad, la asociación entre estudios y emancipación a través de la pareja sigue una pauta en que, a más estudios, mayor probabilidad de convivir en pareja hasta llegar a un máximo entre los que tienen desde unos estudios primarios sin terminar hasta estudios de formación profesional de primer grado; punto álgido en que la tendencia se invierte y se observa como, desde la formación profesional de segundo grado en adelante, a mayor nivel de instrucción, menor probabilidad de formar pareja. No obstante, toda esta pauta se observa por igual entre hombres y entre mujeres, es decir, no se aprecia ningún efecto de género en la relación entre el nivel de instrucción y la convivencia en pareja. La hipótesis que establece que la función del nivel de instrucción sobre la emancipación residencial a través de la pareja es diferencial según género no es cierta en España en el amanecer del siglo XXI. 
Quisiéramos añadir, de nuevo, que no negamos en absoluto que la estructura según el grado educativo de las mujeres jóvenes se elevara en mayor medida que entre los hombres, sino que, al tener en cuenta la edad y la relación con la actividad de una mujer, su probabilidad de estar conviviendo en pareja en lugar de hacerlo con los padres según el nivel de instrucción alcanzado es muy similar entre ellas que entre sus congéneres varones. Por ello, concluimos que la variable género no tiene nada que decir al respecto.

En definitiva, y como conclusiones generales, vemos que, en primer lugar, el peso de la edad en la presión para dejar de convivir con los padres y formar pareja no se manifiesta en España hoy en día hasta edades bastante tardías, y aunque el retraso en la constitución de la unión ha corrido paralelo a un aumento de esta presión, el incremento se da más allá de la etapa biográfica en que se considera enmarcada la juventud, por muy generosa que sea nuestra concepción de esta fase del curso de vida. La formación de la pareja ya ha dejado de ser una dimensión a analizar en la transición de la juventud al mundo adulto.

En segundo lugar, vemos que alcanzar un mayor nivel de instrucción supone alejarse de la formación de la pareja, pero sólo la ausencia de análisis para los varones conduce a la conclusión de que este efecto se da únicamente en las mujeres: para toda la población, independientemente de su sexo, edad y condición laboral, la educación superior en todas las facetas supone una poderosa rémora a la formación de la pareja.

Finalmente, el modelo de formación de pareja con respecto al mundo laboral no ha sufrido ninguna revolución, por mucho que hubiese cambiado la estructura en este aspecto: la mujer que más probabilidad tenía de convivir en pareja era el ama de casa (¡qué remedio!) y, en segundo lugar, la que estaba desocupada, que había abandonado el mercado laboral por las circunstancias que fueran. Esta última pauta no se apreciaba en los varones, los cuales, si estaban parados y habían trabajado con anterioridad, tenían la misma probabilidad de vivir con los padres que en pareja. Esta situación en nada les favorecía ¿por qué si lo hacía entre las mujeres? Las limitaciones de este análisis nos impiden dar cumplida respuesta a esta cuestión, pero sí podemos lanzar una hipótesis: para una mujer, abandonar el mercado de trabajo y situarse en la condición de parada (y tal vez dedicada a la economía doméstica) está mucho más acorde con los tiempos que corren que declararse «ama de casa»; se trata del mismo interior pero con un vestido más apropiado. Por el contrario, resulta curioso que el traje de «estudiante» fuera considerado mucho más decente entre los varones que convivían en pareja y para nada fuese una opción entre las mujeres en pareja. Parecería como si mientras a las mujeres se les ofrecía la posibilidad de o bien declararse sin remilgos como ama de casa o bien afirmarse como paradas, a los hombres sólo se les ofreciera la posibilidad de decir que seguían estudiando. Fuese por lo que fuese, la propensión a convivir en pareja de los varones estudiantes es en la actualidad mayor que entre la población en general, pero las mujeres estudiantes, al igual que las que buscan un primer empleo, están ancladas en una etapa juvenil que rehuye furibundamente el formar pareja, independiente del nivel de instrucción que tengan y de su edad. 


\section{Referencias bibliográficas}

Baizán, P.; Michielin, F. y Billari, F. (2002). «Political economy and lifecourse patterns: The heterogeneity of occupational, family and household trajectories of young Spaniards». Demographic Research, 6, 189-240.

BECKER, G. (1981). Tratado sobre la familia. Madrid: Alianza.

DURÁN, M.A. (dir.) (2000). La contribución del trabajo no remunerado a la economía española: alternativas metodológicas. Madrid: Instituto de la Mujer. (Estudios; 63)

Durkheim, É. (1967). De la división del trabajo social. Buenos Aires: De Schapire.

Jovell, A.J (1995). Análisis de regresión logística. Madrid: CIS. (Cuadernos Metodológicos)

MeIL, G. (1999). La postmodernización de la familia española. Madrid: Acento.

Menacho, T. (2002). Los tipos de estandarización en demografía: Aplicación al estudio de las diferencias regionales de la actividad y desempleo en España, 1990-2000. Memoria de investigación de tercer ciclo. Departament de Geografía. Universitat Autònoma de Barcelona, mimeo.

Miret, P. (2003). «Are Young Europeans less likely to live with a partner as their educational attainment level increases?». Working Papers of the Institute for Social and Economic Researh. Paper 2003-9. Colchester: University of Essex. [http://www.iser.essex.ac.uk/pubs/workpaps/pdf/2003-09.pdf]

- (2007). «Son diferentes las uniones consensuales y los matrimonios? Comparación de los censos españoles de población de 1991 y 2001». Revista Internacional de Sociología, CSIC, LXV (48), 55-74.

Miret, P. y Cabré, A. (2004). "Cohortes decrecientes e inmigración como factor de cambio en los mercados matrimoniales españoles». Revista de Estudios de Juventud. Injuve, 67, 55-70.

- (2005). «Pautas recientes en la formación familiar en España: Constitución de la pareja y fecundidad». Papeles de Economía Española, 104. Número especial sobre transformación demográfica, raíces y consecuencias. Fundación de las Cajas de Ahorros, 17-36.

OPPENHEIMER, V.K. y LEW, V. (1995). «American marriage formation in the 1980s: How important was women's economic independence?». En: OPPENHEIM MASON, K. y JENSEN, A.M. (eds.). Gender and family change in industrialized countries. Oxford: Clarendon Press.

Parsons, T. (1949). "The Social structure of the family». En: Anshen, R. (ed.). The family: its function and destiny. Nueva York: Harper, 172-201.

PARSONS, T. y BALES, R. (1955). Family socialization and interaction process. Glencoe: Free Press. 